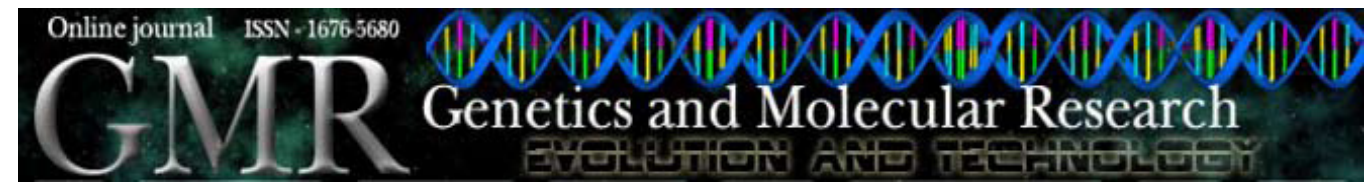

\title{
Expression of Rap1GAP in human myeloid disease following microarray selection
}

\author{
X. Qi, Z. Chen, J. Qian, J. Cen and M. Gu \\ Leukemia Research Division, Jiangsu Institute of Hematology, \\ Key Laboratory of Thrombosis and Hemostasis, Ministry of Health, \\ First Affiliated Hospital, Soochow University, Suzhou, China \\ Corresponding author: Z. Chen \\ E-mail: szchenzx@263.net
}

Genet. Mol. Res. 7 (2): 379-387 (2008)

Received November 25, 2007

Accepted February 15, 2008

Published April 29, 2008

\begin{abstract}
To find the underlying causes of primary myelodysplastic syndrome (MDS), the gene expression profiling of both $\mathrm{CD} 34^{+}$cells and bone marrow mononuclear cells from MDS patients was performed using oligonucleotide microarray and cDNA microarrays, respectively. Several candidate genes which were differentially expressed in MDS patients versus normal controls were selected and confirmed in expanding samples by quantitative real-time reverse transcription-polymerase chain reaction after clustering and bioinformatics analysis. One of these genes, RAP1GAP, was found to be expressed at a significantly higher level in patients with MDS in comparison with those suffering from other hematopoietic diseases including leukemia $(\mathrm{P}<0.01)$. We propose that over-expression of RAP1GAP gene may play a role in the pathogenesis of MDS.
\end{abstract}

Key words: Myelodysplastic syndrome; RAP1GAP; Real-time PCR 


\section{INTRODUCTION}

The myelodysplastic syndromes (MDS) have shared features of refractory cytopenia, dysplastic cellular morphology in the bone marrow and a propensity toward malignant transformation (Gardais, 2000). The variable manifestations and clinical courses are reflected in developing classification schemes based largely on the morphology of bone marrow cells in aspirate smears and in biopsies (Bennett, 2000; Chen et al., 2004). Prognosis is most closely related to the percentages of marrow myeloblasts and also to the presence and character of specific cytogenetic abnormalities (Oosterveld et al., 2003; Chen et al., 2004).

The underlying causes of primary MDS are still being defined. A proposal for the multistep pathogenesis of MDS is that after initial damage to the hematopoietic progenitor cell by a toxin or a spontaneous mutation, several additional alterations may affect these cells and provide them with a growth advantage. These alterations can influence the expression of cell cyclerelated genes, including checkpoint and mismatch repair genes, transcription factors, and tumor suppressor genes. In addition, early MDS has been associated with an elevated ratio of apoptosis to proliferation (Gardais, 2000), but the mechanisms for this finding are not yet established.

The defect of the hematopoietic stem cell in MDS is not well characterized. One technical problem is that most of the experiments that use clinical samples from MDS patients have been performed with low-density, non-adherent cells from the bone marrow of these patients. This may be adequate for high-risk MDS and acute myeloid leukemia (AML) developed from MDS because of the more uniform blast population in the bone marrow. In contrast, with low-risk MDS, bone marrow is extremely heterogeneous and hypocellular. Therefore, molecular abnormalities characteristic of malignant cells are more difficult to find in low-risk MDS than in high-risk MDS or AML (Hofmann et al., 2002). Purification of malignant cells by cell sorting or laser capture microdissection has the advantage of yielding a more homogeneous population of cells for study but has the disadvantage of increasing the processing time and extent of tissue manipulation. While nonmalignant components of tumors are often considered "contaminating", it is likely that such nonmalignant cells carry important information regarding the pathogenesis of the malignancy in question (Ebert and Golub, 2004). Lee et al., 2001 demonstrated patterns of normal and dysplasia-related transcription among unpurified bone marrow mononuclear cells (BMNCs) and generated relevant gene transcription patterns from heterogeneous marrow cell populations, suggesting that it may be usefully applied to complex malignant cell populations (Lee et al., 2001; Ebert and Golub, 2004). In the present study, we first obtained a gene expression profile from $\mathrm{CD} 34^{+}$cells using oligonucleotide microarray, and then used cDNA microarrays to confirm these results in BMNCs of MDS patients (Qian et al., 2005a). We also extended our findings by determining the expression level of Rap1GAP, one of the candidate genes selected by bioinformatics analysis, in MDS and other myeloid diseases.

\section{PATIENTS, MATERIAL AND METHODS}

\section{Patients}

$\mathrm{CD}_{4} 4^{+}$cells isolated from 8 samples including 2 refractory anemia (RA), 1 RA with ringed sideroblast (RAS), 2 RA with excess of blasts (RAEB), 2 RA with excess of blasts in transformation (RAEBt), and 1 healthy control (Table 1) were applied to microarray hybridization for gene expression profiling. BMNCs selected from 9 MDS patients (5 males, 4 females) 
and 4 healthy controls were studied at initial diagnosis (Table 2). Patients were classified as RA or RAEB according to the French-American-British Cooperative Group criteria.

\begin{tabular}{lccclc}
\multicolumn{6}{l}{ Table 1. Clinical characteristics of the patients whose CD34 } \\
\hline Patient & Age (years) & Gender & FAB type & \multicolumn{1}{c}{ Karyotype } & IPSS \\
\hline RAEBt 1 & 34 & Male & RAEBt & $46, \mathrm{XY}$, tandem duplication(1)(q12q24) & High \\
RAEBt 2 & 70 & Female & RAEBt & $47, \mathrm{XX},+8$ & High \\
RAEB 1 & 43 & Male & RAEB & $43, \mathrm{XY}, 5 \mathrm{q}-, 6 \mathrm{p}+,-7,-\operatorname{der}(12) \mathrm{t}(12 ; 18)$ & Int-2 \\
& & & & $(\mathrm{q} 11 ; \mathrm{q} 23), \operatorname{dic}(15 ; 21), \operatorname{der}(18) \mathrm{t}(12 ; 18)[8]$ & \\
RAEB 2 & 40 & Female & RAEB & $46, \mathrm{XX}, \operatorname{der}(6)[3] / 47$, idem, $+8[7]$ & Int-2 \\
RA 1 & 53 & Female & RA & RA/47,XX, +8,9q-[4]/48,idem, +der(1)[7]/ & Int-2 \\
& & & & $48,+\operatorname{der}(1), 9 \mathrm{q}-,+9 \mathrm{q}-,+$ mar[CP2]/46,XX[2] & \\
RA 2 & 40 & Male & RA & $44, \mathrm{XY}, \operatorname{del}(5)(\mathrm{q} 12 \mathrm{q} 31),-7,-18$ & Int-2 \\
RAS & 51 & Male & RAS & $45, \mathrm{XY},-5,-6,+$ mar & Int-1 \\
\hline
\end{tabular}

$\overline{\mathrm{FAB}}=$ French-American-British Cooperative Group criteria; $\mathrm{RA}=$ refractory anemia; RAS $=$ RA with ringed sideroblast; RAEB = RA with excess of blasts; RAEBt = RA with excess of blasts in transformation.

Table 2. Clinical characteristics of the patients whose bone marrow mononuclear cells were detected by microar-
rays.
\begin{tabular}{lccccc}
\hline Case & Gender & Age (years) & FAB type & Karyotype & IPSS \\
\hline 1 & Male & 29 & RAEB & $46, \mathrm{XY}$ & Int- 1 \\
3 & Female & 30 & RAEB & $46, \mathrm{XX}$ & Int- 1 \\
4 & Male & 52 & RAEB & $45, \mathrm{XY},-7, \mathrm{i}(20 \mathrm{q}-)$ & Int- \\
5 & Male & 50 & RAEB & $47, \mathrm{XY},+8$ & High \\
6 & Female & 65 & RA & $46, \mathrm{XX}$ & Int- 1 \\
7 & Female & 34 & RAEBt & $46, \mathrm{XX}$ & High \\
8 & Male & 44 & RA & $46, \mathrm{XY}$ & Int-1 \\
9 & Male & 36 & RA & $46, \mathrm{XY}, 20 \mathrm{q}-$ & Int- 1 \\
10 & Female & 48 & RA & $47, \mathrm{XX},+8$ & Int- 1 \\
\hline For abbreviations, see legend to Table 1. & & &
\end{tabular}

\section{Sample preparation}

Heparinized bone marrow samples were obtained by aspiration from the posterior iliac crest after written informed consent from all patients. To lessen activation of the cells by any technical manipulation, fresh bone marrow was processed immediately after aspiration to select the mononuclear cells within the subsequent $4 \mathrm{~h}$. CD $34^{+}$cells were purified according to the protocol (Hofmann et al., 2002). Mononuclear cells were separated by density gradient centrifugation through Ficoll-Hypaque. Total RNA was extracted using TRIzol (Invitrogen, Shanghai, China) according to the manufacturer protocol with minor modifications.

\section{Oligonucleotide microarray, cDNA microarray and data analysis}

Eight pieces of Human Genome U133 Plus 2.0 Array were used. Seven chips were hybridized with amplified products from 7 patients and 1 healthy control. A detailed protocol for the sample preparation and microarray processing is available from Affymetrix (Santa Clara, CA, USA). Since there was a very small amount of cells from which the RNAs could be extracted, two rounds of in vitro transcript amplification were needed before hybridization on the oligonucleotide microarray. 
cDNA microarray chips were purchased from Biostar Company (United Gene Holdings Co. Ltd., Shanghai, China). In every chip, 500 potential genes involved in hematopoiesis regulation were printed in quadruplicate. Microarray analysis was performed for individual samples.

GeneChip image analysis was performed using the Microarray Analysis Suites version 5.0 (Affymetrix). Data analysis was performed with the GeneSpring software version 6.0 (Silicon Genetics, San Carlos, CA, USA). The samples derived from patients were analyzed independently with comparison to appropriate healthy control. Samples were normalized for expression levels in each chip to reference values. Statistical analyses of the average expression level (analysis of variance, ANOVA) were then carried out for all individual genes in the test samples from the MDS patients, based on comparison with healthy control. Fold changes for the log ratios are shown. The signal $\log 2$ ratio $\geq 1$ was considered as "up", whereas the signal $\log 2$ ratio $\leq-1$ as "down". Patterns of gene expression were identified using a hierarchical-clustering algorithm as a gene tree or a condition tree to indicate related expression. Nomenclature and functional descriptions were derived from public databases.

\section{Real-time quantitative RT-PCR}

Quantification of RNA in bone marrow cells using real-time reverse transcriptionpolymerase chain reaction (RT-PCR) was performed as described previously. Briefly, $500 \mathrm{ng}$ total RNA was processed directly to cDNA by RT. PCR primers and Taqman probes (Table 3) were designed using the software Primer Express 2.0 with published sequence data from the NCBI database. Amplification reactions contained $2 \mu \mathrm{L}$ cDNA, $1 \mu \mathrm{L} 20 \mathrm{X}$ buffer, $2 \mu \mathrm{L} \mathrm{MgCL}_{2}$, $4 \mu \mathrm{L} 5 \mathrm{X}$ Q, $0.8 \mathrm{mM}$ dNTPs, $2 \mu \mathrm{M}$ primers, $1 \mu \mathrm{M}$ probes, $1 \mathrm{U}$ Hotstar polymerase (QIAGEN, Germany) in a final volume of $20 \mu \mathrm{L}$. The thermal cycling conditions were as follows: $15 \mathrm{~min}$ at $95^{\circ} \mathrm{C}$, followed by 40 cycles at $95^{\circ} \mathrm{C}$ for $30 \mathrm{~s}, 50^{\circ} \mathrm{C}$ for $30 \mathrm{~s}$, and $72^{\circ} \mathrm{C}$ for $30 \mathrm{~s}$. $\beta$-actin was used as an active and endogenous reference to correct for differences in the amount of total RNA added to a reaction and to compensate for different levels of inhibition during reverse transcription of RNA and during PCR. Each sample was replicated three times. Nonparametric tests in SPSS were used for statistical analyses.

\begin{tabular}{lll}
\multicolumn{2}{l}{ Table 3. Sequence of primers and probes. } \\
\hline Gene & Rap1GAP & \\
\hline Primer-F & 5'-CAAGAin \\
Primer-R & 5'-GCCACGTGCTATAGAGAGACC-3' & 5'-TCACCCACACTGTGCCCATCTACGA-3' \\
Probe & 5'-FAM-AGCAGAGGCGCTCAA & 5'-CAGCGGAACCGCTCATTGCCAATGG-3' \\
& GGACTTCTCC-TAMRA-3' & 5'-FAM-ATGCCCTCCCCATGCCATCC \\
\hline
\end{tabular}

\section{RESULTS}

\section{Gene expression profile analysis in patients with MDS}

After normalization, 18,404 dots of signal on the Affymetrix chips were filtered for the final clustering and bioinformatics searching in CD34 ${ }^{+}$cell profiles (Figure 1A). RAP1 GTPase activating protein 1 (Rap1GAP), one of the genes thought to be involved in hematopoietic regulation, was in the up-regulated group. As shown in Figure 1A, the two "RA" and the "RAS" showed upregulation of Rap1GAP. Based on these results, we next determined the gene expression profiles of 
BMNCs from MDS and healthy subjects using a spotted cDNA microarray. Genes were selected only if four spots for each individual gene were all effective in one chip. Furthermore, only genes demonstrating a similar expression pattern in all four spots, either up-regulated or down-regulated in the same subtype of patients could be chosen for further analysis. A total of 39 of the 500 genes potentially involved in hematopoiesis regulation, cell growth, and cell cycle control and signaling were selected for final analysis. Among them, 18 genes including ets variant gene 1 (ETV1) and Rap1GAP were in the up-regulated group and 21 genes in the down-regulated group including DDIT3 (Qian et al., 2005b) (Table 4, Figure 1B). In this cohort of MDS patients examined for BMNCs, Rap1GAP (also called RAP1GAP) also belonged to the up-regulated group. Since the expression pattern of this gene in MDS patients detected in both $\mathrm{CD} 34^{+}$bone marrow cells and BMNCs using different kinds of microarray was in concordance, we will concentrate on the expression pattern of this gene in more patients with MDS and other human myeloid diseases.
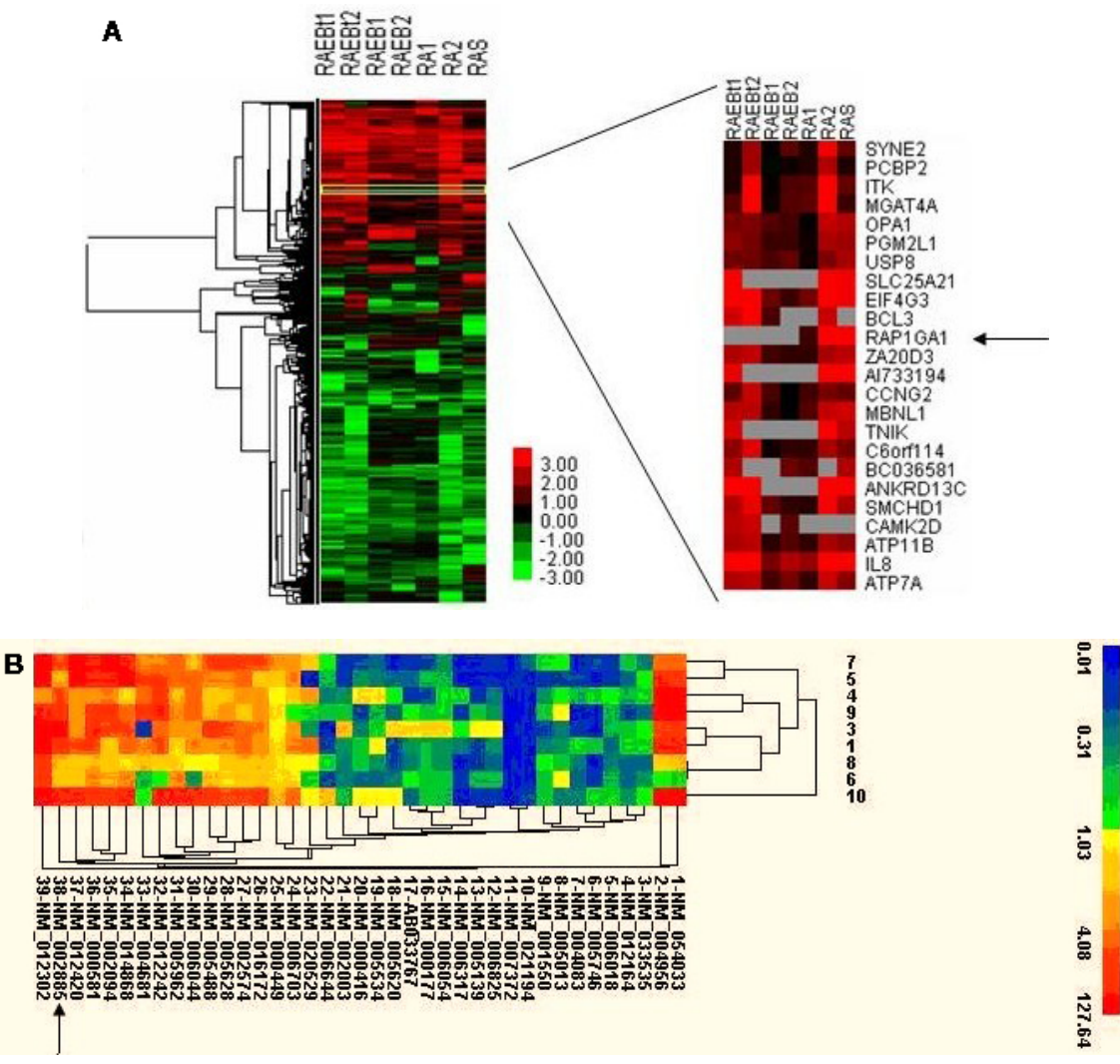

Figure 1. Identification of genes expressed in $\mathrm{CD} 34^{+}$cells (A) and bone marrow mononuclear cells (B) can be distinguished between patients and healthy controls. Each row represents a separate patient sample, and each column a single gene on the microarray. The clustering is presented graphically as a colored image. Along the horizontal axis, the analyzed genes are arranged as ordered by the clustering algorithm. The genes with the most similar patterns of expression are placed adjacent to each other. The color and intensity of each cell in the image represent the expression level of each gene, with red representing an expression level higher than the mean, green representing an expression level lower than the mean, and the brighter color intensity represents a greater magnitude of deviation from the mean. Rap1GAP (RAP1GA1) is marked by an arrow. For abbreviations, see legend to Table 1 . 
Table 4. Abnormal gene expression pattern detected in bone marrow mononuclear cells from patients with myelodysplastic syndrome.

\begin{tabular}{|c|c|c|c|}
\hline Gene & Accession & Map & Description \\
\hline FKBP1B & NM_054033 & $2 \mathrm{p} 23.3$ & FK506 binding protein $1 \mathrm{~B}$ \\
\hline ETV1 & NM_004956 & $7 \mathrm{p} 21.3$ & ets variant gene 1 \\
\hline RAP1GAP & NM_002885 & $1 \mathrm{p} 36.1-\mathrm{p} 35$ & RAP1, GTPase-activating protein 1 \\
\hline RNF10 & NM_014868 & $12 \mathrm{q} 24.31$ & ring finger protein 10 \\
\hline GPX1 & NM_000581 & $3 \mathrm{p} 21.3$ & glutathione peroxidase 1 \\
\hline FBXL5 & NM_033535 & $4 p 15.33$ & F-box and leucine-rich repeat protein 5 \\
\hline FBXW2 & NM_012164 & $9 \mathrm{q} 34$ & F-box and WD-40 domain protein 2 \\
\hline HDAC6 & NM_006044 & $\mathrm{Xp} 11.23$ & histone deacetylase 6 \\
\hline SLC30A1 & NM_021194 & $1 \mathrm{q} 32-\mathrm{q} 41$ & zinc transporter 1 \\
\hline DDX42 & NM_007372 & $17 \mathrm{q} 23.3$ & DEAD box polypeptide 42 protein \\
\hline PBEF1 & NM_005746 & $7 \mathrm{q} 22.3$ & pre-B-cell colony-enhancing factor \\
\hline CKAP4 & NM_006825 & $12 \mathrm{q} 23.3$ & transmembrane protein $(63 \mathrm{kDa})$ \\
\hline FCN1 & NM_002003 & $9 q 34$ & ficolin 1 \\
\hline S100A11 & NM_005620 & 1q21 & S100 calcium-binding protein A11 \\
\hline ANXA3 & NM_005139 & $4 q 13-q 22$ & Annexin III \\
\hline HSPH1 & NM_006644 & $13 q 12.3$ & heat shock $105-\mathrm{kDa}$ protein 1 \\
\hline BASP1 & NM_006317 & $5 \mathrm{p} 15.1-\mathrm{p} 14$ & brain acid-soluble protein 1 \\
\hline IFNGR2 & NM_005534 & $21 \mathrm{q} 22.11$ & interferon gamma receptor factor-1 \\
\hline GSN & NM_000177 & $9 q 33$ & Gelsolin \\
\hline NUCB2 & NM_005013 & $11 \mathrm{p} 15.1-\mathrm{p} 14$ & HGNC:8044, NEFA \\
\hline $\mathrm{BSCv}$ & AB-̄033767 & $20 \mathrm{p} 11.22-\mathrm{p} 11.21$ & C20orf3 \\
\hline DDIT3 & NM_004083 & $12 \mathrm{q} 13.1-\mathrm{q} 13.2$ & C/EBP homologous protein \\
\hline RTN3 & NM_006054 & $11 \mathrm{q} 13$ & ASY interacting protein \\
\hline GPR109B & NM_006018 & $12 \mathrm{q} 24.31$ & GTP-binding protein \\
\hline IFRD1 & NM_001550 & $7 q 22-q 31$ & nerve factor-inducible protein $\mathrm{PC} 4$ \\
\hline IFNGR1 & NM_000416 & $6 \mathrm{q} 23-\mathrm{q} 24$ & Immune interferon, receptor for \\
\hline RFX5 & NM_000449 & $1 \mathrm{q} 21$ & regulatory factor $\mathrm{X}, 5$ \\
\hline NFKBIA & NM_020529 & $14 \mathrm{q} 13$ & IkappaBalpha \\
\hline EIF1AY & NM_004681 & Yq11.222 & translation initiation factor $1 \mathrm{~A}$ \\
\hline NUDT3 & NM_006703 & $6 \mathrm{p} 21.2$ & nudix-type motif 3 \\
\hline DKK1 & NM_012242 & $10 \mathrm{q} 11.2$ & dickkopf homolog 1 \\
\hline GSPT1 & NM_002094 & $16 \mathrm{p} 13.1$ & G1 to $\mathrm{S}$ phase transition protein \\
\hline UBADC1 & NM_016172 & $9 q 34.3$ & Ubiquitin associated domain containing 1 \\
\hline PRDX1 & NM_002574 & $1 \mathrm{p} 34.1$ & natural killer-enhancing factor $\mathrm{A}$ \\
\hline SLC1A5 & NM_005628 & $19 \mathrm{q} 13.3$ & RD114 virus receptor \\
\hline TOM1 & NM_005488 & $22 \mathrm{q} 13.1$ & target of myb 1 \\
\hline MXI1 & NM_005962 & $10 q 24-q 25$ & MAX interacting protein 1 \\
\hline IFIT5 & NM_012420 & $10 \mathrm{q} 23.31$ & retinoic acid and interferon-inducible protein \\
\hline LPHN2 & NM_012302 & $1 \mathrm{p} 31.1$ & latrophilin 2 \\
\hline
\end{tabular}

\section{Detection of Rap1GAP expression level by real-time quantitative RT-PCR}

To confirm the preferential expression of the RAP1GAP gene in MDS BMNCs, we prepared cDNAs from samples of 33 patients with MDS (14 RA, 6 RAS, 13 RAEB, and RAEBt), 8 with AML, and 8 with non-hematologic malignancies as controls. These cDNAs were subjected to real-time quantitative PCR analysis with primers specific for Rap1GAP and $\beta$-actin.

The median levels of Rap1GAP transcripts in patients with MDS, AML, and nonmalignant blood diseases (including 1 pure megakaryotic anemia, 4 iron deficiency anemia, 2 hypercellular anemia, 1 idiopathic thrombocytopenia) were 0.084 (ranging from 0.0005 to 0.54 ), 0.0015 (ranging from 0 to 0.0036 ), and 0.0113 (ranging from 0 to 0.0558 ), respectively 
(Figure 2). The abundance of Rap1GAP mRNA relative to that of $\beta$-actin mRNA in the cells from most MDS patients was significantly greater than that in the BMNCs from most AML and non-malignant blood diseases $(\mathrm{P}<0.01)$. Among RA, RAS and non-malignant blood diseases, differences were also shown $(\mathrm{P}<0.05)$, while no statistically significant difference could be found between AML and non-malignant blood diseases $(\mathrm{P}>0.05)$. Each sample was replicated three times. Nonparametric tests in SPSS were used for statistical analyses.

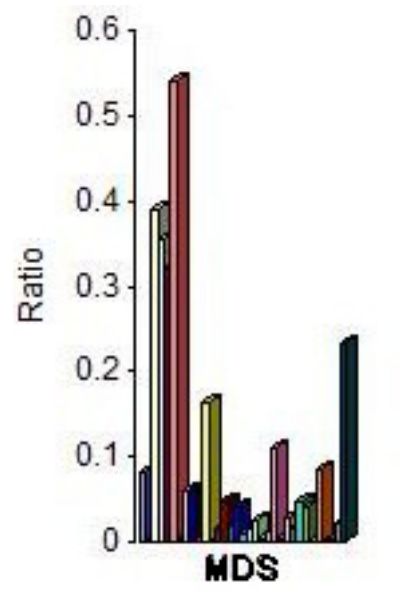

\section{Rap1GAP/ $\beta$-actin}

Figure 2. Quantitation of Rap1GAP mRNA in the bone marrow mononuclear cell bank samples from patients with myelodysplastic syndrome (MDS), acute myeloid leukemia (AML), or non-malignant blood diseases. The cDNA prepared from the blasts of 33 patients with MDS (14 RA, 6 RAS, 13 RAEB, and RAEBt), 8 with AML, and 8 with non-malignant blood diseases (1 pure megakaryotic anemia, 4 iron deficiency anemia, 2 hypercellular anemia, 1 idiopathic thrombocytopenia) was subjected to real-time PCR with primers specific for Rap1GAP or $\beta$-actin. The ratio of the abundance of Rap1GAP transcripts to that of $\beta$-actin transcripts (Rap1GAP/ $\beta$-actin) was calculated for statistical analysis $(\mathrm{p}<0.01)$.

\section{DISCUSSION}

Oncogenic mutations of ras genes are detected in up to $30 \%$ of human malignancies, but they are rare in some type of cancers, including leukemia. In an attempt to identify the genes that could counteract the action of oncogenic ras genes (Kometani et al., 2004), Kitayama et al. (1989) identified a gene called Rap1 (Ras-proximate-1). Rap1, a small G protein in the Ras superfamily, is involved in signal transduction cascades. It is highly homologous to Ras, but is down-regulated by its own set of GAPs (GTPase-activating proteins) (Brinkmann et al., 2002). Rap1 is implicated in the regulation of a variety of cellular processes (Delehanty et al., 2003; Bivona et al., 2004; Chen et al., 2005), including the control of platelet activation (Delehanty et al., 2003; Kometani et al., 2004; Schultess et al., 2005; Philip and Tara, 2005), T-cell anergy (Ishida et al., 2003; Bivona et al., 2004), B-cell activation (Kometani et al., 2004; Philip and Tara, 2005), and neuronal differentiation (Kometani et al., 2004; Chen et al., 2005). Rap1 was shown to inhibit K-Ras-mediated transformation as well as the growth factor-induced and Ras-mediated mitogen-activated protein kinase activation. In contrast to its suggested role as a 
Ras antagonist, Rap1 becomes activated along with Ras by receptor tyrosine kinase activators and is believed to mediate its own type of intracellular signals (Brinkmann et al., 2002). Rap1 has received recent attention for its role in enhancing integrin-dependent signals. This action of Rap1 augments a variety of processes that characterize hematopoietic-cell function, including aggregation, migration, extravasations, and homing to target tissues. Recent studies indicate that Rap1 is involved in the maturation of megakaryocytes, the pathogenesis of chronic myelogenous leukemia, and the activation of peripheral T cells (Philip and Tara, 2005). Like all G proteins, Rap1 exists in an inactive guanine nucleotide diphosphate (GDP)-bound state and is activated when GDP is exchanged for guanine nucleotide triphosphate (GTP). Like Ras, it cycles between a GDP-bound inactive and a GTP-bound active form, and this switching is regulated by specific guanine nucleotide exchange factors (GEFs) and GAPs (Philip and Tara, 2005).

Rap1GAP, the first RapGAP, unlike RapGEFs, as it has no sequence homology with RasGAP and other GAPs such as RanGAP or RhoGAP (Brinkmann et al., 2002; Daumke et al., 2004). Rap1GAP was originally identified on plasma membranes isolated from differentiated promyelocytic HL-60 cells (Philip and Tara, 2005), and is expressed at a low level in proliferating cells which increase upon differentiation (Tsygankova et al., 2004). The cytosolic form of Rap1GAP worked on Rap1 and had no activity toward Ras or any other small GTP-binding proteins. Recent results propose an interaction between Rap1GAP and the Gai isoform of heterotrimeric G proteins (Mochizuki et al., 1999), and similar connections to Gaz and Gao have also been reported (Kometani et al., 2004). Inactive Gao binds to and sequesters Rap1GAP, whereas activated Gaz recruits Rap1GAP to the membrane. In both cases, receptor-mediated activation of the heterotrimeric $G$ proteins results in the attenuation of Rap 1 activation on the membrane. It is suggested that Rap1GAP has a crucial role in gating the signal flow from Rap1 to its downstream effectors at the cell surface or the cytoskeleton (Kometani et al., 2004).

Although for the first time we have found that Rap1GAP is differentially expressed in MDS compared to other hematopoietic diseases including leukemia, and have raised the issue that Rap1GAP may play a role in the pathogenesis of MDS, much remains to be verified on the exact mechanism. Why and how the abundance of Rap1GAP mRNA in the cells from most MDS patients is markedly greater than that in the BMNCs from most AML and other patients and whether the expression levels correlate with the prognosis of MDS patients also need to be explored.

\section{ACKNOWLEDGMENTS}

Research supported by grant \#30470733 from the National Natural Scientific Foundation of China, grant \#BK2004040 from the Natural Scientific Foundation of Provincial Scientific Bureau of Jiangsu, and grant \#135XY0407 from 135 open grant of Jiangsu Provincial Scientific Bureau.

\section{REFERENCES}

Bennett JM (2000). World Health Organization classification of the acute leukemias and myelodysplastic syndrome. Int. J. Hematol. 72: 131-133.

Bivona TG, Wiener HH, Ahearn IM, Silletti J, et al. (2004). Rap1 up-regulation and activation on plasma membrane regulates T cell adhesion. J. Cell Biol. 164: 461-470.

Brinkmann T, Daumke O, Herbrand U, Kuhlmann D, et al. (2002). Rap-specific GTPase activating protein follows an 
alternative mechanism. J. Biol. Chem. 277: 12525-12531.

Chen G, Zeng W, Miyazato A, Billings E, et al. (2004). Distinctive gene expression profiles of CD34 cells from patients with myelodysplastic syndrome characterized by specific chromosomal abnormalities. Blood 104: 4210-4218.

Chen Y, Wang PY and Ghosh A (2005). Regulation of cortical dendrite development by Rap1 signaling. Mol. Cell Neurosci. 28: 215-228.

Daumke O, Weyand M, Chakrabarti PP, Vetter IR, et al. (2004). The GTPase-activating protein Rap1GAP uses a catalytic asparagine. Nature 429: 197-201.

Delehanty LL, Mogass M, Gonias SL, Racke FK, et al. (2003). Stromal inhibition of megakaryocytic differentiation is associated with blockade of sustained Rap1 activation. Blood 101: 1744-1751.

Ebert BL and Golub TR (2004). Genomic approaches to hematologic malignancies. Blood 104: 923-932.

Gardais J (2000). Dyshaemopoiesis in adults: a practical classification for diagnosis and management. Leuk. Res. 24: 641-651.

Hofmann WK, de Vos S, Komor M, Hoelzer D, et al. (2002). Characterization of gene expression of CD34 cells from normal and myelodysplastic bone marrow. Blood 100: 3553-3560.

Ishida D, Yang H, Masuda K, Uesugi K, et al. (2003). Antigen-driven T cell anergy and defective memory T cell response via deregulated Rap1 activation in SPA-1-deficient mice. P. N. A. S. 100: 10919-10924.

Kitayama H, Sugimoto Y, Matsuzaki T, Ikawa Y, et al. (1989). A Ras-related gene with transformation suppressor activity. Cell 56: 77-84.

Kometani K, Ishida D, Hattori M, Minato N (2004). Rap1 and SPA-1 in hematologic malignancy. Trends Mol. Med. 10: 401-408

Lee YT, Miller LD, Gubin AN, Makhlouf F, et al. (2001). Transcription patterning of uncoupled proliferation and differentiation in myelodysplastic bone marrow with erythroid-focused arrays. Blood 98: 1914-1921.

Mochizuki N, Ohba Y, Kiyokawa E, Kurata T, et al. (1999). Activation of the ERK/MAPK pathway by an isoform of rap1GAP associated with G alpha(i). Nature 400: 891-894.

Oosterveld M, Wittebol SH, Lemmens WA, Kiemeney BA et al. (2003). The impact of intensive antileukaemic treatment strategies on prognosis of myelodysplastic syndrome patients aged less than 61 years according to International Prognostic Scoring System Risk Groups. Br. J. Haematol. 123: 81-89.

Philip JSS and Tara JD (2005). Multiple roles of Rap1 in hematopoietic cells: complementary versus antagonistic functions. Blood 106: 2952-2961.

Qian J, Chen Z, Wang W, Cen J, et al. (2005a). Gene expression profiling of the bone marrow mononuclear cells from patients with myelodysplastic syndrome. Oncol. Rep. 14: 1189-1197.

Qian J, Chen Z, Lin J, Wang W, et al. (2005b). Decreased expression of CCAAT/enhancer binding protein zeta (C/ EBPzeta) in patients with different myeloid diseases. Leuk. Res. 29: 1435-1441.

Schultess J, Danielewski O and Smolenski AP (2005). Rap1GAP2 is a new GTPase-activating protein of Rap1 expressed in human platelets. Blood 105: 3185-3192.

Tsygankova OM, Feshchenko E, Klein PS and Meinkoth JL (2004). Thyroid-stimulating hormone/cAMP and glycogen synthase kinase 3beta elicit opposing effects on Rap1GAP stability. J. Biol. Chem. 279: 5501-5507. 